\title{
Naufragentes in hoc mari - Zur Symbolik des Wassers in Berichten über die Seereise des HI. Brandan
}

\begin{abstract}
The sea voyage, especially through open-water, was considered one of the most dangerous modes of travel during the Middle Ages due to unpredictable forces of nature and the numerous marvels (MHG wunder) that could be expected. This article explores medieval literary texts devoted to the seafaring Saint Brendan (German Brandan), focusing on the various forms and meanings of the sea and of water as well as their semantic shifts over time. For instance, the notion of the sea as a symbolic space filled with salvific history prevails in the Latin 'Navigatio sancti Brendani abbatis', initially written for a monastic audience. The exemplary abbot Brendan can be viewed as having transcended the perils of this space. By contrast, a further concept emerges in vernacular versions, especially in the German 'Reise'. Coincidence (contingentia) seems to dominate providence (providentia) within the narrative manifested in the repentant sinner Brendan who occasionally even fears the wonders and force of the sea.
\end{abstract}

Keywords: ,Navigatio sancti Brendani abbatis‘, ,Reise‘, Retextualisierung, Symbolik, Wasser

\section{Brandan und die mappae mundi}

Die Vita des irischen Heiligen Brandan, der im 6. Jahrhundert lebte und wirkte, ${ }^{1}$ unterscheidet sich nicht maßgeblich von der anderer Heiliger. Was ihm aber schon recht bald nach seinem Dahinscheiden eine Ausnahmestellung in diesem erlesenen Kreis garantierte, sind seine Seereisen. Ich möchte mich im Zusammenhang mit dem Wasser, dessen Unerschöpflichkeit als „Reservoir kultureller Symbolwelten“ Hartmut BöHmE betont, ${ }^{2}$ in der Hauptsache auf den Aspekt des Meeres beschränken. Folgt

1 Vgl. Clara Strijbosch, The Seafaring Saint. Sources and Analogues of the Twelfth Century Voyage of Saint Brendan, Dublin 2000, S. 1. Weiterführende Forschungsliteratur zur Biographie Brandans findet sich ebd. in Anm. 3.

2 Hartmut BöHME, Umriss einer Kulturgeschichte des Wassers. Eine Einleitung, in: DERs. (Hg.), Kulturgeschichte des Wassers, Frankfurt / M. 1988, S. 7-42, hier S. 13. An anderer Stelle meint BöHME: „Unzählig die Beziehungen, die die Kulturen real und symbolisch mit dem Wasser unterhalten“ (ebd., S. 15); „Der Symbol- und Zeichengebrauch des Wassers wirkt bestimmend im Aberglauben wie in der Schöpfungstheologie, in der Brunnenkur wie der Taufe, im Mythos und der Lyrik, im Abenteuerroman wie im Seemannsgarn, in volksliterarischen Überlieferungen wie in metapoetologischen Erzählungen, 
man dem frühen Erzählzweig der ,Navigatio sancti Brendani abbatis ‘, ${ }^{3}$ führt Brandan eine seiner Meerfahrten bis zur sogenannten terra repromissionis sanctorum. Dabei segelt er mit einer ausgewählten Gruppe von Mönchen teilweise wochenlang über das offene Meer, bevor er wieder auf eine Insel trifft - etwas, das man in der realhistorisch belegten Seefahrt möglichst zu vermeiden suchte. ${ }^{4}$ Insgesamt sieben Jahre müssen die Mönche reisen, ehe sie die Insel erreichen. Dabei folgt die Reise zyklisch dem Kirchenjahr, sie verbringen die wichtigsten liturgischen Feste an immer denselben Orten. ${ }^{5}$ Auf ihrem Weg erleben sie viele Wunder - gefährliche wie gnadenreiche. Daher erstaunt es nicht, dass die Insel des Brandan auf den mappae mundi des Mittelalters, wie etwa der Hereford-Karte oder der Ebstorfer Weltkarte, am Rand der Ökumene verortet wird. Es ist auf diesen Karten vor allem der äußere Ring, in dem Orte mit überwiegend utopischer Qualität zu finden sind, nicht zuletzt auch das irdische Paradies, das in der Stoff- und Motivtradition oft mit der terra repromissionis sanctorum überblendet wird. ${ }^{6}$ Auch die Topoi des Schreckens, wo unreine Völker und Fabelwesen leben, sind an den

im spontanen Traumbild wie in artifiziellen Wasserlandschaften, in der Wassermusik wie im Märchen, im Tempelritus und der Gartenbaukunst, in der Magie wie in der Psychoanalyse, in Sagen über Wassermonstren wie im heiligen Text über das Einwohnen Gottes im Wasser“ (ebd., S. 19). Einen Schnitt durch die Zeiten und Kulturen zum Thema des Wassers und seiner Symbolik liefert die äußerst verdienstvolle Monographie von Karl Matthäus Woschiтz, Fons Vitae - Lebensquell. Sinn- und Symbolgeschichte des Wassers (Forschungen zur europäischen Geistesgeschichte 3), Freiburg / Br., Basel, Wien 2003.

3 Knappe Übersichten zu den einzelnen Erzählzweigen finden sich $u$. a. bei Clara STRIJBosch, Searching for a Versatile Saint. Introduction, in: Glyn S. BuRgess u. Clara STRIJBosch (Hgg.), The Brendan Legend. Texts and Versions (The Northern World 24), Leiden, Boston 2006, S. 1-9; Glyn S. BuRgESS, Introduction. The Life and Legend of Saint Brendan, in: The Voyage of St Brendan. Representative Versions of the Legend in English Translation with Indexes of Themes and Motifs from the Stories, hrsg. v. William R. J. BARRoN u. Glyn S. Burgess, Exeter 2005, S. 1-12. Zu überlieferten Textzeugen, Editionen und Übersetzungen (bis zum Jahr 2000) s. Glyn S. BuRgess u. Clara STRIJBosch, The Legend of St Brendan. A Critical Bibliography, Dublin 2000, S. 1-103.

4 Vgl. Norbert OHLER, Reisen im Mittelalter, München, Zürich 1986, S. 64. Dort heißt es: „Die theoretisch mögliche große Geschwindigkeit der Schiffe wurde fast nie erreicht. Denn bis in die Neuzeit fuhr man aus Sicherheits- und Bequemlichkeitsgründen nicht den idealen Kurs, sondern in der Küstennähe bzw. von Insel zu Insel [...]. Bei drohendem Sturm wollte man sich in den Schutz eines Hafens, mindestens in den Windschatten einer Insel oder eines Kaps flüchten. An Land fand man frisches Trinkwasser, Nahrung und Feuerholz.“ Dass Reisen in Küstennähe bevorzugt wurden, belegen die zahlreichen Portolankarten, die in ihrer Darstellung auf die originalgetreue und detailreiche Abbildung von Küstenabschnitten spezialisiert waren. Die notwendige Engführung von Realgeschichte und Kulturgeschichte des Wassers begründet BöHmE (Anm. 2), S. 19-20.

5 Damit begegnet die Erzählung dem naheliegenden Vorwurf, dass die Mönche gegen das benediktinische Gebot der stabilitas loci verstoßen, vgl. hierzu auch Simone LoLEIT, Ritual und Augenschein. $\mathrm{Zu}$ Gedächtnis und Erinnerung in den deutschen Übersetzungen der ,Navigatio Sancti Brendani ‘ und der deutsch-niederländischen Überlieferung der ,Reise'-Fassung (Essener Beiträge zur Kulturgeschichte 3), Aachen 2003, passim.

6 Vgl. Hartmut Kugler, Der ,Alexanderroman` und die literarische Universalgeographie, in: Udo Schöning (Hg.), Internationalität nationaler Literaturen. Beiträge zum ersten Symposion des Göttinger Sonderforschungsbereichs 529, Göttingen 2000, S. 102-120, hier S. 114. 
Peripherien der Ökumene zu finden. Aber alles wird von Gott umfasst und gehalten, wie es die Ebstorfer Karte durch die am Rand des orbis terrae abgebildeten Glieder Jesu (Kopf, Hände, Füße) symbolisiert. Gott ist Schöpfer und Gestalter - artifex und creator mundi. Und dieser ständige Bezug von Kosmologie, Heilsgeschichte und Topographie - ein Signum mittelalterlicher Weltkarten schlechthin - ist auch der ,Navigatio‘ eingeschrieben.

\section{Das Meer als Allegorie und die ,Navigatio}

Das Meer ist zunächst einmal im sensus litteralis ein gefährlicher Ort. Wer es befährt, muss der mittelalterlichen Enzyklopädie folgend nicht nur mit jeder Menge Fabelwesen, sondern auch mit enormen Naturgewalten rechnen. Um diese lebend zu überstehen, wandte man sich an eben jenen Gott, der auch das Weltmeer auf der Ebstorfer Karte umfasst. So berichtet Beda Venerabilis aus der Mitte des 7. Jahrhunderts, „während eines Seesturms hätten Reisende heiliges Öl, das ihnen Bischof Aidan in Erwartung des Unwetters mitgegeben hatte, auf die Wogen gegossen; daraufhin habe sich gleich der Wind gelegt". ${ }^{7}$ Selbst Kolumbus, der sich mit dem größten nautischen Know-how seiner Zeit auf die Amerikafahrten begab, weiß sich in einem Orkan keinen anderen Rat, als eine Wallfahrt zu geloben. ${ }^{8}$

Dieses „Vertrauen in Gottes Hilfe“, ${ }^{9}$ das neben den Instrumenten und der Erfahrung des Steuermanns unabdingbar war, inszeniert die ,Navigatio“ literarisch, und zwar in einem monastisch geprägten Umfeld. So geht sie über den sensus litteralis hinaus und lässt eine Deutung des Meeres im Sinne der mittelalterlichen Hermeneutik mit ihrem vierfachen Schriftsinn zu. Derart lässt sich die Reise Brandans mit Walter HAUG als sieben Jahre währende „Fahrt durch das Meer des Lebens zur himmlischen Heimat“ lesen. ${ }^{10}$ Diese Allegorie der Schifffahrt hat eine lange Tradition. ${ }^{11}$ Schon Columban hat dieses Bild für seine Predigten genutzt: „Columbanus, in one of his sermons, makes use of the image of the spiritual ship and the dangers at

7 OHLER (Anm. 4), S. 69.

8 Ebd. Auch Vasco da Gama wendet sich in der Not auf See an transzendente Mächte: „Wir waren an einem Punkt angekommen, an dem alle Manneszucht aufhörte. Während wir so in der Todesnot weiterfuhren, taten wir auf den Schiffen viele Gelübde an Heilige und Fürsprecher“ (ebd., S. 74-75).

9 Ebd., S. 67.

10 Zuerst erschienen in der Reihe „Wolfram-Studien“: Walter HAUG, Vom Imram zur Aventiure-Fahrt, in: Werner SCHRöDER (Hg.), Wolfram-Studien 1, Berlin 1970, S. 264-298. Hier und im Folgenden wird nach der erneuten Veröffentlichung zitiert: Walter HAUG, Vom Imram zur Aventiure-Fahrt, in: DERS., Strukturen als Schlüssel zur Welt. Kleine Schriften zur Erzählliteratur des Mittelalters, Tübingen 1989, S. 379-407, hier S. 399.

11 Vgl. Heimo Reinitzer, Wasser des Todes und Wasser des Lebens. Über den geistigen Sinn des Wassers im Mittelalter, in: BöHmE, Kulturgeschichte des Wassers (Anm. 2), S.99-144; Dietrich SchmidTKE, Geistliche Schiffahrt. Zum Thema des Schiffes der Buße im Spätmittelalter, in: Beiträge 
sea to the believer in Christ, and as Christ as our Pilot (recalling the walk upon the sea of Galilee)“. ${ }^{12}$ Entsprechend heißt es auch im ,Hymnus sancti Brendani confessoris“ aus dem 11. Jahrhundert: Is imploret nos salvari / naufragentes in hoc mari, / ferat opem cito lapsis / pressis mole gravis fascis. ${ }^{13}$

Für die irischen Mönche der ,Navigatio“ ist der Ozean gleichbedeutend mit der Wüste als Ort der Abgeschiedenheit, womit sie sich in die Tradition der frühchristlichen Eremiten stellen. ${ }^{14}$ Diese semantische Verknüpfung von Anachoretentum und Meer über die Analogie von Meer und Wüste behält selbst einige Jahrhunderte nach Entstehung der ,Navigatio“ ihre Aktualität. In der frühneuhochdeutschen Übersetzung des Kartäusers Heinrich Haller um die Mitte des 15. Jahrhunderts ist in der Vorrede zu lesen:

Dÿe nachgeschriben hystorÿ vnd sach dye ist sagen von der wandrung vnd pilgramschaft des heyligen wrendani vnd von den grossen wunderwerchen dye got der herr durch jn gewarcht hat vnd wie er jn genërt vnd gespeyst hat syben jar mit seinen prüedern jn der wilden wüest vnd auf dem vngestuemen mer occeano vnd wye er jn pehuett hat mit seinen prüedernn vor den posen geysten vnd vor den wildenn tyernn vnd vor den graussamen merwundernn $[. ..] .^{15}$

wüest und mer erscheinen hier nicht als komplementäre Ausdrücke, sondern sie sind als semantisch deckungsgleich $\mathrm{zu}$ verstehen, gewissermaßen eine Zwillingsformel wie wandrung vnd pilgramschaft oder genërt vnd gespeyst. ${ }^{16}$ Die Deklaration der See-

zur Geschichte der deutschen Sprache und Literatur 91 (1969), S. 357-385, und 92 (1970), S. 115-177; auch von Hugo RAHNER sind einige grundlegende Arbeiten zu diesem Thema erschienen.

12 Dorothy Anne BraY, Allegory in the ,Navigatio Sancti Brendani', in: Viator 26 (1995), S. 1-10, hier S. 2 .

13 Original zitiert nach Ernst DÜMMLER, Gedichte aus Ivrea, in: Zeitschrift für deutsches Alterthum 14 (1896), S. 245-265, hier S. 256. Der Schiffbruch im Sinne einer Daseinsmetapher ist seit der Antike ein immer wiederkehrendes Bild, vgl. Hans BLuMENBERG, Schiffbruch mit Zuschauer. Paradigma einer Daseinsmetapher (Suhrkamp-Taschenbuch Wissenschaft 289), Frankfurt / M. 1979; vgl. auch BöHME (Anm. 2), S. 26. Schon im ,Hexaemeron' des Ambrosius findet sich am Ende einer längeren Ausführung zum Meer das folgende Gebet, das auch den Schiffbruch des Glaubens mit einbezieht, vor dem er bewahrt werden möchte: Det nobis Dominus illa sucessuum flumina prospero ligno currere, tuto portu consistere, nequitiae spiritalis graviora quam ferre possumus, tentamenta nescire, fidei ignorare naufragia, habere pacem profundam: et si quando aliquid sit, quod graves nobis saeculi hujus excitet fluctus, evigilantem pro nobis habere gubernatorem Dominum Jesum, qui verbo imperet, tempestatem mitiget, tranquilitatem maris refundat, cui est honor et gloria, laus, perpetuitas a saeculis et nunc et semper, in omnia saecula saeculorum. Amen, Original zitiert nach REINITZER (Anm. 11), S. 135, Anm. 12.

14 Vgl. Reinhold Grimm, Paradisus coelestis, Paradisus terrestris. Zur Auslegungsgeschichte des Paradieses im Abendland bis um 1200 (Medium Aevum 33), München 1977, S. 106.

15 Zitiert nach: Sankt Brandans Meerfahrt. Ein lateinischer Text und seine drei deutschen Übertragungen aus dem 15. Jahrhundert, hrsg. v. Karl A. ZAENKER (Stuttgarter Arbeiten zur Germanistik 191), Stuttgart 1987, S. 3 unten, Z. 2-7.

16 Vgl. Christoph FASBEndER, Brandan-Probleme. Eine Nachlese zur Neuausgabe, in: Amsterdamer Beiträge zur älteren Germanistik 56 (2002), S. 103-122, hier S. 121. Vom Prinzip her drücken sich in diesen beiden Erscheinungsformen die Extreme von absolutem Wassermangel einerseits und größ- 
reise als Pilgerfahrt befördert eine rein geistliche Lesart des Textes - genauso wie die Zuschreibung von Speisewundern und dämonischen Angriffen zum Meeresraum. Doch das alles Entscheidende ist auch hier: Das Meer ist der genuine Wirk- und Herrschaftsraum Gottes, in dessen Verfügungsgewalt sich der Seereisende begibt. Sobald Brandan in See sticht, wirkt Gott durch ihn Wunder und hält ihn am Leben, indem er ihn speist und vor allem Unheil beschützt.

Was Jean Delumeau für die kollektive Mentalität des Mittelalters behauptet, nämlich dass das Meer als „Ort der Angst, des Todes und des Wahnsinns“ betrachtet wurde, ${ }^{17}$ gilt nun gerade nicht für den Brandan der ,Navigatio‘. In den zahlreichen Begegnungen mit den Naturgewalten und Dämonen des Meeres erweist sich der Heilige als besonders mutig und garantiert das ein oder andere Mal durch rationale Entscheidungen sein Überleben und das seiner ihm unterstehenden Mönche. Als sie beispielsweise auf der Insel anlanden, die eigentlich ein riesiger Fisch ist, bleibt Brandan an Bord des Schiffes (uir Dei sedebat intus in naui ${ }^{18}$ ). Er weiß, was es mit der Insel auf sich hat, möchte seine Begleiter aber nicht ängstigen (ne perterreren$\left.t^{19}{ }^{19}\right)$. Am nächsten Morgen wollen sich die Mönche über einem Feuer Essen zubereiten, da beginnt der Fisch langsam zu versinken. Es ist Brandan, der durch seine Voraussicht die anderen rettet, indem er jeden einzelnen von ihnen an der Hand in das Schiff zieht (At ille singulos per manus trahebat intus [sc. in naui] ${ }^{20}$ ). Wie sich zeigt, ist das Meer für Brandan zudem nicht der sichere Weg in den Tod, sondern eine Bewährung und ein erster Schritt dahin, den Tod in der imitatio Christi zu bezwingen. Dass er die alltagsbezogene, profane Bedeutung des Meeres als „Ort der Angst, des Todes und des Wahnsinns“ (s. o.) in seiner Heiligkeit komplett negiert, darf freilich nicht verwundern. Er verkörpert in der Tugend der providentia den göttlichen Heilsplan, erhält Weisung und befolgt sie anstandslos. ${ }^{21}$ Er ist damit gewissermaßen ein ,Wasser-Heros‘ wie Odysseus oder Kolumbus, sein Männerbild gründet dagegen

tem Überfluss an Wasser andererseits aus, vgl. BöHME (Anm. 2), S. 13, wie sie beispielsweise das Volk Israel im Alten Testament innerhalb kurzer Zeit am eigenen Leibe spürt, vgl. ebd., S. 27-28. Aber auch, wenn man eine Übersetzung „öde Gegend, Wildnis“ für wüest veranschlagt, ist die Analogie zum Meer als andersartige Einöde und Wildnis - man denke nur an die zur Formel geronnene Nominalphrase wilde see - schlagend.

17 Jean Delumeau, Angst im Abendland. Die Geschichte kollektiver Ängste im Europa des 14. bis 18. Jahrhunderts, Bd. 1, Reinbek / Hamburg 1985, S. 61.

18 Lateinisches Original hier und im Folgenden zitiert nach: Navigatio Sancti Brendani Abbatis, hrsg. v. Carl Selmer, Notre Dame / Ind. 1959, hier Kap. 10, S. 20, Z. 7.

19 Ebd., Kap. 10, S. 20, Z. 8.

20 Ebd., Kap. 10, S. 21, Z. 16-17.

21 Es darf allerdings nicht verschwiegen werden, dass die Erzählung diesbezüglich eine Differenz zwischen dem Abt und dem Rest der Gruppe etabliert, vgl. Brigitte STARK, Terra repromissionis Sanctorum. Die Reise des Heiligen Brendan zum irdischen Paradies, in: Jan A. AERTSEN u. Andreas SPEER (Hgg.), Raum und Raumvorstellungen im Mittelalter (Miscellanea mediaevalia 25), Berlin 1998, S. 525-539, hier S. 530. 
nicht auf thalassalen Kampf- und Eroberungsdynamiken, ${ }^{22}$ sondern auf christlicher Demut und Ergebenheit.

\section{Brandan als Sünder (,Reise'-Fassung)}

Etwas anders ist es um den Brandan der ,Reise'-Fassung aus der Mitte des 12. Jahrhunderts bestellt. Für ihn ist die Seereise deutlich als via purgativa gezeichnet, denn er hat sich versündigt, indem er ein Buch verbrannte, in welchem von den Wundern Gottes die Rede ist: er enwolde noch enmachte / des icht geloubic wesen, / wie er ez hette gelesen, / er ensehez mit den ougen sin. ${ }^{23}$ Damit ähnelt er dem ungläubigen Thomas, mit dem er in der ,Reise'-Fassung explizit verglichen wird. Das Meer wird zum Raum der Überzeugung durch die adtestatio rei visae Brandans, denn er bekommt von einem Engel den Auftrag, die Wunder selbst zu sehen und das verbrannte Buch neu zu schreiben (Fassung N). Das Novum ist hier „die Spannung zwischen Glauben und Wirklichkeit““. ${ }^{24}$ Brandan steht in der ,Reise'-Fassung mehr als Mensch denn als Heiliger „einer Welt gegenüber, deren wunderbarer, d. h. auf Gott bezogener Charakter der Enthüllung bedarf“. ${ }^{25}$ Es ist die Reise selbst, die ihn in seinem Glauben stärkt und heiligt, wie Julia WeItBREcht in ihrer Dissertation dargelegt hat. ${ }^{26}$ Damit unterscheidet sich die Art der Bewährung grundlegend von der der ,Navigatio“ - das Meer wird zum „Raum von Wandel und Buße“. ${ }^{27}$ Die Reise wird, wenn auch nicht genealogisch, so doch phänomenologisch zur Zwischenstufe zwischen der ,Navigatio“ und dem Aventiureroman, wie Walter HAUg aufzeigen konnte. ${ }^{28}$ Das Abenteuer, das der Artusritter im Wald sucht, bestimmt auch die Reise Brandans zur See. Wenn man so will, stehen sich hier festländischer und thalassaler Kontingenzraum gegenüber. ${ }^{29}$ Es ist sehr auffällig, wie oft sich Brandan oder eine andere Figur der Erzählung in der

22 Vgl. BöHme (Anm. 2), S. 26.

23 Ich zitiere hier und im Folgenden - sofern nicht anders angegeben - die Fassung M, und zwar nach: Brandan. Die mitteldeutsche ,Reise'-Fassung, hrsg. v. Reinhard HAHN u. Christoph FASBENDER (Jenaer germanistische Forschungen, N. F. 14), Heidelberg 2002, V. 44-47.

24 HAUG (Anm. 10), S. 403.

25 Ebd.

26 Vgl. Julia Weiтвrecht, Aus der Welt. Reise und Heiligung in Legenden und Jenseitsreisen der Spätantike und des Mittelalters (Beiträge zur älteren Literaturgeschichte), Heidelberg 2011.

27 Ebd., S. 203.

28 Vgl. Haug (Anm. 10), S. 403-405.

29 Vgl. Mireille Schnyder, Räume der Kontingenz, in: Cornelia Herberichs u. Susanne ReICHLIN (Hgg.), Kein Zufall. Konzeptionen von Kontingenz in der mittelalterlichen Literatur (Historische Semantik 13), Göttingen 2010, S. 174-185. Für die ,Reise'-Fassung spricht auch Weitbrecht (Anm. 26), S. 203, von Kontingenz. 
,Reise‘-Fassung M dezidiert versigelt, d. h. sich segelnd verirrt. ${ }^{30}$ Zudem wird die feste, dem Kirchenjahr folgende Stationenfolge der ,Navigatio“ hier vollkommen aufgehoben, was den Eindruck des ziellosen Umhertreibens noch zusätzlich verstärkt.

Mit dieser Kontingenz, die als Ausdruck archaischer Chaotik des Urwassers angesehen werden kann, ${ }^{31}$ geht dann in der ,Reise'-Fassung eine Unsicherheit auf Seiten Brandans einher: uf dem abgrunde er swebete / in vil grozer ungewisheit. / were gote sine vart leit, / so were ez im gewesen ungewerlich. ${ }^{32}$ Teilweise ist es auch pure Angst: do brachte sie in sorgen / ein visch, der was vreisan. / vor dem kiele er stete ran, / er wolde da verslinden den kiel / [...] in wart ein angest vil groz. ${ }^{33}$ Für den Brandan der ,Reise'-Fassung wird das Meer damit immer wieder zu einem locus horribilis. ${ }^{34}$ Der irische Abt ist, wie WEITBREcHT feststellt, „gerade kein Heiliger voller Gottvertrauen, dem nichts etwas anhaben kann“, sein „harmonisches Verhältnis zur Transzendenz ist im Gegenteil [...] massiv gestört und soll im Verlauf der Reise wiederhergestellt werden“. ${ }^{35}$ In der ,Reise'-Fassung geht er denn auch mit den anderen zusammen auf den Riesenfisch und wird genau wie sie vom Hinabsinken des Fisches überrascht: einen durren boum sie vunden. / do sie den houwen begunden, / do gienc daz wilde lant / sin wec hin alzuhant, / daz der vil heilige man / den kiel kume wider gewan. ${ }^{36}$ Erneut hat Brandan große Angst: der getruwe sente Brandan / was in vil engestlicher not. ${ }^{37}$ Allerdings erscheint diese Angst funktionalisiert, denn es heißt weiter:

30 So etwa Hahn u. Fasbender (Anm. 23) in den Versen 285, 295, 300, 402, 630 etc.; in V. 246-247 heißt es in einer Anthropomorphisierung der Wellen, welche Brandan zu einem Objekt macht, das dem Willen des Wassers unterworfen scheint: do namen sie die unde / und slugen sie in ein lant; ähnlich V. 310: do begonde sie ein wint triben.

31 Vgl. BöHme (Anm. 2), S. 28.

32 HAHN u. FASBENDER (Anm. 23), V. 932-935.

33 Ebd., V. 1420-1427.

34 Hier lassen sich in nuce auch mentalitätsgeschichtliche Zusammenhänge über das Wasser herstellen, wie sie BöHME (Anm. 2), S. 24, für die kulturgeschichtliche Forschung zum Wasser einfordert: es reiche nicht hin, nur die Motiv- und Imaginationstypen des Wassers zu erfassen, z. B. das Leben als Schifffahrt, diese müssten vielmehr mit der Bildungsgeschichte von Subjektivität, insbesondere von Gefühls- und Imaginationsformen verbunden werden.

35 WeitBrecht (Anm. 26), S. 204. Dazu auch Loleit (Anm. 5), S. 71: „Daß Brandan das Ordnungssystem, in dem er sich befindet, nicht durchschaut, kommt im Bild der geleiteten Reise zum Ausdruck: Nicht Brandan ist der ,Steuermann', sondern das Spiel von Wind und Wellen als Instrumentarium des göttlichen Willens.“ Vgl. außerdem ebd., S. 143, Anm. 313; Helga NeumanN, Reden über Gott. Brandans Meerfahrt - Diskursdifferenzierung im 15. Jahrhundert, in: Jan-Dirk MüLLER u. Horst WENZEL (Hgg.), Mittelalter. Neue Wege durch einen alten Kontinent, Stuttgart 1999, S. 181-196, besonders S. 192.

36 HAHN u. FASBENDER (Anm. 23), V. 179-184.

37 Ebd., V. 206-207. 
sus giengez im als got gebot. / daz tet got allez um daz, / daz erz geloubete deste baz, / daz daz buch die warheit saite. / alrest er do daz claite, / daz got nicht ungerochen liez, / daz er daz buch verburnen hiez. / er gedachte in sinem mute / , herre got du gute, / swer dir missetruwet, / wie liechte ez in geruwet! [...] $]^{\text {38 }}$

Da er den Fisch aufgrund dessen, was er im Buch gelesen hat, im Nachhinein durchaus als Wunder Gottes zu deuten weiß, ist seine Angst eine Angst vor Gott und damit wiederum eine Tugend. ${ }^{39}$ Sie verhilft ihm zur Einsicht, zur Reue, womit das Meer über seine furchterregenden Bewohner zum Raum der Belehrung, aber auch zum Gedächtnisort im Sinne der ars memorativa wird. ${ }^{40}$

Zudem fällt beim Vergleich dieser Episode in der ,Navigatio'-Fassung mit der ,Reise'-Fassung die wiederholt betonte Bewaldung des Fisches in letzterer auf, wo das Tier in der ,Navigatio‘ anfangs noch als steinige Insel ohne Grasbewuchs wahrgenommen wird: Erat autem illa insula petrosa sine ulla herba ${ }^{41}$ Zeichenhaft werden hier die unterschiedlichen Realisierungsmöglichkeiten der Kontingenzräume - der undurchdringliche und keinen Horizont erkennen lassende Wald und das nur durch den Horizont begrenzte Meer - überblendet und dadurch verstärkt. ${ }^{42}$

Pauschal zu behaupten, dass die ,Reise'-Fassung von Kontingenz geprägt ist, griffe jedoch etwas zu kurz. Denn bei näherer Betrachtung fällt auf, dass der Eindruck des Zufalls entweder durch die Wahrnehmung der Figuren entsteht oder durch die Zuschreibung des Erzählers erfolgt. Eine absolute Kontingenz kann es in der mittelalterlichen Literatur nicht geben - oder um mit Mireille SCHNYDER zu sprechen: „[D]ie perspektivenlose, verwirrende Not ist Teil eines göttlichen Plans.“43 Kontingenz findet immer im Rahmen der Providenz statt, sie ergibt sich immer nur teilperspektivisch aus der defizitären Wahrnehmung einer Figur heraus. ${ }^{44}$ Die räumliche und zeit-

38 Ebd., V. 208-218.

39 Vgl. Peter Dinzelbacher, Einleitung, in: Ders., Angst im Mittelalter. Teufels-, Todes- und Gotteserfahrung. Mentalitätsgeschichte und Ikonographie, Paderborn 1996, S. 9-25, hier S. 16. Vgl. auch Hartmut BöHme, Himmel und Hölle als Gefühlsräume, in: Claudia BenthIEN, Anne FlEIG u. Ingrid KAsten (Hgg.), Emotionalität. Zur Geschichte der Gefühle (Literatur - Kultur - Geschlecht. Kleine Reihe 16), Köln 2000, S. 60-81.

40 Vgl. in diesem Zusammenhang LoleIt (Anm. 5) zur lectio und meditatio.

41 Selmer (Anm. 18), Kap. 10, S. 20, Z. 5.

$42 \mathrm{Zu}$ der unmessbaren Weite des Raumes kommt eine vergleichsweise lange Zeit hinzu, die die Mönche auf dem Meer - und nicht auf Inseln - verbringen. Das ist ein Zug, der in der ,Navigatio“ durch die vermehrte Angabe von Zeiträumen besonders betont wird; dazu LoLEIT (Anm. 5), S. 31: „Das Meer ist nicht nur unübersehbar weit, sondern die Etappen der Fahrt sind zudem, im Vergleich mit den Aufenthalten auf den Inseln, extrem lang. Dadurch wird die Zeit, die die Mönche auf dem Meer verbringen, als ebenso unüberschaubar dargestellt wie der Raum.“

43 SCHNyder (Anm. 29), S. 177.

44 Vgl. bezüglich des Brandanstoffs WeItBRecht (Anm. 26), S. 204, oder Udo FriedRICH, Anfang und Ende. Die Paradieserzählung als kulturelles Narrativ in der ,Brandanlegende‘ und im ,Erec ‘ Hartmanns von Aue, in: Ders., Andreas Hammer u. Christiane Witthöft (Hgg.), Anfang und Ende. For- 
liche Ordnung Gottes, der ordo, kann schlichtweg nicht außer Kraft gesetzt werden. ${ }^{45}$ Das Meer ist und bleibt damit immer ein Ort der Gefahr, der aber, wenn man ihn als Raum göttlichen Waltens im sensus spiritualis begreift, transzendiert werden kann. Dann ist er immer noch notwendig, aber nicht mehr notwendig gefährlich.

Warum nun dieser Wandel der Brandanfigur und ihres Verständnisses vom Raum des Meeres? Das mag sicherlich in den wechselnden Umständen der Rezeption gründen, denen sich der Text in seinen verschiedenen Retextualisierungen anpassen muss, um das Identifikationspotential mit seinem Protagonisten aufrechtzuerhalten. In einem genuin monastischen Umfeld von Religiosen, in dem die lateinische ,Navigatio' firmierte, wird Brandan vor allem als idealer Abt, als imitator Christi gezeichnet. Sobald die Geschichte - dann zumeist in Form der Volkssprache - in die Grenzräume zur monastischen Laiengemeinschaft (Konversen) bzw. zur höfischen Kultur eindringt, muss Brandan als ihr Handlungsträger offenbar modifiziert - und das heißt zumeist: menschlicher - werden.

\section{4 ,Orientierung' auf dem Meer}

Dass das Meer ein Raum der Symbolik ist, den unterschiedliche Kulturkreise anders ausdeuten, kann auch an den Himmelsrichtungen und der Verortung bestimmter Jenseitsstätten aufgezeigt werden. Viele der lateinischen Handschriften der ,Navigatio“ verlegen das irdische Paradies in den Westen, was die Forschung durch den Einfluss der irischen Mythologie auf das dortige Christentum erklärt hat. Nach jener Vorstellung gibt es „eine paradiesische ,Insel der Verheißung“ (tir tairngire), die gegen Sonnenuntergang im Ozean liegt “ ${ }^{46}$ Das widerspricht nun aber der kanonischen Ansicht, wie sie beispielsweise einige Alexanderromane in Form der ,Iter ad paradisum'-Episode exemplifizieren. Demnach kann das irdische Paradies nur im Osten, also Richtung Orient, zu finden sein. Und so verwundert es kaum, dass Brandan spätestens in

men narrativer Zeitmodellierung in der Vormoderne (Literatur - Theorie - Geschichte 3), Berlin 2014, S. 267-288, hier S. 276: „Die scheinbar zufällige Aggregation der Stationen wird durch die Koordinaten der Heilsgeschichte zusammengehalten.“

45 Vgl. hierzu die Prosafassung der ,Reise“ ( $\mathrm{Ph}=$ Heidelberg, Universitätsbibliothek, Cpg 60, fol. 174r), als sie multum bona terra erreichen: $v$ nd das selb land haiszet jn der hailligen gschrifft Bona terra vnd lit ver von der welt. wann nun das gott wolte, das sÿ das mer dar trüg, sÿ mo̊chten sunst nit dar sin kómen vn die verhengnusz gotz. Die direkte Gewalt über das Meer wird in einer weiteren Prosafassung der ,Reise' (Pm = München, Universitätsbibliothek, $2^{\circ}$ Cod. Ms. 688, fol. 250r) noch mehr betont: vnd es lit ferre von diser welt. wann das got wolt, $d z$ sy darin kemend vnd liesz sy das mer dar tragen, Sy mochtent sunst nit da hin komen sin, wann $d z$ es got wolt.

46 ZAENKER (Anm. 15), S. xi. Vgl. auch Andreas HAMmER, St. Brandan und das ander paradîse, in: Kathryn Starkey u. Horst Wenzel (Hgg.), Imagination und Deixis. Studien zur Wahrnehmung im Mittelalter, Stuttgart 2007, S. 153-176, hier S. 165. 
den Übersetzungen der ,Navigatio oftmals gen Osten segelt, ${ }^{47}$ wie bei Johannes Hartlieb (kurz vor 1450): so wellenn wir faren gein orient $z w$ ainer insel die haist daz gelobt lannd der heiligen, ${ }^{48}$ und Heinrich Haller (nach 1450): sy hetenn ainen grossen weyten weg czu varen gegen dem land orient, ${ }^{49}$ oder die Richtungsangabe Westen komplett wegfällt, wie im Falle von ,Der Hilligen Levent.$^{50}$ Die ,Orientierung‘ des Paradieses ist auch in anderen volkssprachlichen Fassungen $\mathrm{zu}$ beobachten, so beispielsweise in der anglo-normannischen des ,Benedeit' (Anfang 12. Jahrhundert), ${ }^{51}$ der des ,South English Legendary“ (ca. 1300) ${ }^{52}$ oder der venezianischen (14. Jahrhundert). ${ }^{53}$ Dabei treten die Änderungen zwar selten konsequent über den ganzen Text hindurch auf, aber es wird doch deutlich genug, dass sie absichtsvoll vorgenommen wurden. ${ }^{54}$ Das erscheint plausibel, wenn man die primäre Funktion der Himmelsrichtungen mit Karl ZAENKER darin sieht, ,auf den verborgenen sensus allegoricus in der Erzählung hinzuweisen“. ${ }^{55}$ Diese Referenzfunktion der Himmelsrichtungen ist hingegen in der ,Reise'-Fassung M nicht auszumachen. Angaben zur Verortung der beiden irdischen Paradiese sind nicht zu finden. Sofern überhaupt Richtungsangaben existieren, wirken sie vielmehr arbiträr. Generell „sind die wenigen Bestimmungen von Zeit und Raum [...] zumeist relativ: die Ereignisse verweisen aufeinander [...], nicht jedoch [...] auf den jenseits der Welt stehenden Gott“ ${ }^{56}$ was den bereits besprochenen Eindruck von Kontingenz in der ,Reise‘-Fassung zusätzlich untermauert.

\section{Quellen, Brunnen, Flüsse, Seen}

Im Zusammenhang mit dem irdischen Paradies bzw. den irdischen Paradiesen - in der ,Reise'-Fassung gibt es zwei - rückt die Erzählung jedoch einen weiteren Aspekt des Wassers in den Vordergrund, der hier zumindest noch angedeutet werden soll: die Differenz vom Meer als Salzwasser einerseits und Quellen, Brunnen, Flüssen und

47 Tendenzen zur ,Um-Orientierung' sind aber auch schon in einigen lateinischen Handschriften auszumachen, vgl. ZAENKER (Anm. 15), S. xii.

48 ZAENKER (Anm. 15), S. 10 unten, Z. 4-5.

49 Ebd., S. 147 unten, Z. 11-12. Haller ist dabei nicht ganz konsequent, vgl. ebd., S. xii.

50 Vgl. ebd., S. xi.

51 Tendent lur curs vers orïent („They steer their course towards the east“), zitiert nach: The AngloNorman Voyage of St Brendan. Bilingual Edition, hrsg. v. Jude S. MACKLEY, Northampton 2013, V. 1641. 52 Rizot euene toward pen est fourti dawas hi wende, zitiert nach BARRON u. BURGESS (Anm. 3), S. 319, V. 683.

53 „Let us sail towards the east, that we may go to the island which is called the Promised Land of the Saints“, hier die englische Übersetzung zitiert nach BARRoN u. BURGESS (Anm. 3), S. 165. Vgl. dazu auch die Begründung durch Mark DAviE, ebd., S. 156.

54 Vgl. etwa ZaEnKer (Anm. 15), S. xi.

55 Ebd., S. xiii.

56 Neumann (Anm. 35), S. 190. 
Seen als Süßwasser andererseits. Dies ist unter anderen eine Differenz, die sich in der Patristik als eine von Wasser des Todes und Wasser des Lebens wiederfindet. ${ }^{57}$ Für die ,Navigatio‘, wo sich auf den Inseln immer wieder Quellen, Brunnen und Flüsse finden, hat Simone LoLEIT festgestellt: „Deren Wasser kann als Metonymie für das Wasser der Paradiesflüsse gelesen werden und somit als lebendiges Wasser, das dem Meer als Ort von Sünde und Tod entgegensteht. “58 Diese Elemente, die in der ,Navigatio“ symbolisch auf das Sakrament der Taufe verweisen, ${ }^{59}$ finden sich allerdings auch in der ,Reise'-Fassung wieder. So fällt auf, dass Brandan über einen Fluss zum ersten irdischen Paradies gelangt: vur sente Brandan / bie einem wazzer zu tale / kegen dem aller schonstem sale, / den ie kein ouge gesach. / darzu truc sie des wazzers bach. ${ }^{60}$ Der Fluss ist, genau wie die dort herrschende Finsternis, als ein typisches Schwellenelement auf dem Weg zu einem heiligen Raum zu sehen. ${ }^{61}$ Womöglich ist der Fluss sogar als Phison, also einer der Paradiesflüsse zu denken, denn Ambrosius meinte, „man könne durch ihn leicht zum Paradies zurückkehren (per hanc facile ad paradisum revertatur)“ ${ }^{62}$ Über den Phison heißt es bei Ambrosius weiter: Nomen est uni Phison; hic est qui circuit omnem terram Euilat, ubi est aurum. terrae autem illius aurum bonum est, ubi est carbunculus, et lapis prasinus. ${ }^{63}$ Auch der Fluss, auf dem Brandan zum Paradies fährt, weist einen goldenen und mit vielen Edelsteinen übersäten Grund auf. ${ }^{64}$ Bei der Rückkehr sammeln Brandan und seine Brüder viel des Goldes und der Edelsteine ein, um es später für den schmückenden Bau von Kirchen zu nutzen. Inwiefern hier die exegetische Literatur mit ihrer allegorischen Ausdeutung dieser res mitschwingt, kann kaum geklärt werden. ${ }^{65}$ Insgesamt scheint die Ähnlichkeit zur ,Iter ad paradisum‘-Episode etwa im ,Straßburger Alexander‘ auffällig. Auch Alexan-

57 Vgl. dazu grundlegend REINITZER (Anm. 11).

58 Loleit (Anm. 5), S. 33.

59 Vgl. ebd., S. 32.

60 HAHN u. FASBENDER (Anm. 23), V. 472-476.

61 Hammer (Anm. 46), S. 162. Für HAMmeR zeigen allein schon „die Gefahren der Seereise [die] verdeutlichte Schwellensituation beim Übergang an“, s. ebd. Dies gilt freilich auch für die „Anderswelt“ der keltischen Mythologie, mit der die Brandan-Materia historisch verflochten ist, vgl. ebd., S. 165.

62 Grimm (Anm. 14), S. 122; vielleicht sei das aber allegorisch zu verstehen, s. ebd.

63 Ambrosius Mediolanensis, Opera pars prima qua continentur libri: Exameron, De paradiso, De Cain et Abel, De Noe, De Abraham, De Isaac, De bono mortis, hrsg. v. Karl Schenkl (Corpus scriptorum ecclesiasticorum Latinorum 32,1), Prag, Wien, Leipzig 1897, ,De paradiso', III, 14. In Gen 2, 11-13 lauten die Informationen und Formulierungen bezüglich des Phison fast identisch, vom carbunculus oder lapis prasinus jedoch ist nicht die Rede: nomen uni Phison ipse est qui circuit omnem terram Evilat ubi nascitur aurum et aurum terrae illius optimum est ibique invenitur bdellium et lapis onychinus, zitiert nach: Biblia Sacra. Iuxta vulgatam versionem, hrsg. v. Roger GRYson, Stuttgart 2007.

64 HAHN u. FASBENDER (Anm. 23), V. 459-465, V. 565-572.

65 Bei Ambrosius verweise das Gold auf die klugen Entdeckungen (pro inventis prudentibus), der glänzende carbunculus sei dann der Feuerschein der Seele; das Gold bedeute bei Augustinus die disciplina vivendi, der Karfunkel wiederum die veritas, die durch keine Nacht verdunkelt werde, s. GRIMM (Anm. 14), S. 126-127. 
der reist auf einem der Paradiesflüsse, dem Euphrat, zum irdischen Paradies. ${ }^{66}$ Dabei handelt es sich bei der Benennung des Flusses im ,Straßburger Alexander um eine signifikante Änderung, da in der recht festen ,Iter ad paradisum'-Tradition eigentlich der Phison anstelle des Euphrats steht. ${ }^{67}$ Genau wie in der ,Reise-Fassung $M$ ist das Paradies dann mit einer Mauer umgeben, die die finale Grenze für die Reisenden darstellt. Die Tatsache, dass sich Brandan in absoluter Dunkelheit auf dem Fluss gen irdisches Paradies bewegt, könnte wiederum durch die Annahme des Augustinus, dass „die Paradiesesflüsse streckenweise unterirdisch verliefen“, ${ }^{68}$ beeinflusst sein. ${ }^{69}$

\section{Schluss}

Es konnte kursorisch aufgezeigt werden, dass das Brandan-Corpus in seinen unterschiedlichen literarischen Ausformungen an der reichhaltigen Symboltradition des Wassers teilhat - vor allem an der patristisch geprägten. Die unterschiedlichen Eigen-

66 Vgl. Peter StRohschneIDER u. Herfried VöGEL, Flußübergänge. Zur Komposition des ,Straßburger Alexander', in: Zeitschrift für deutsches Altertum und deutsche Literatur 118 (1989), S. 85-108; Markus Sтоск, Kombinationssinn. Narrative Strukturexperimente im ,Straßburger Alexander', im ,Herzog Ernst B‘ und im ,König Rother (Münchener Texte und Untersuchungen zur deutschen Literatur des Mittelalters 123), Tübingen 2002. „[S]ehr im Widerspruch zu den vier Paradiesesflüssen“ steht für GRIMM (Anm. 14), S. 110, der Fluss auf der terra repromissionis sanctorum in der ,Navigatio'. Er teilt die Insel und hindert Brandan daran, weiter in das Paradies vorzudringen, womit er eine ähnliche Funktion wie die Paradiesmauer des ersten irdischen Paradieses in der ,Reise‘-Fassung erfüllt.

67 Vgl. Sтоск (Anm. 66), S. 131.

68 GRIMm (Anm. 14), S. 122.

69 So heißt es auch auf der Ebstorfer Weltkarte zum Paradies (direkt unter dem Kopf Jesu): In hoc fons oritur, qui in IIII flumina divitur, que quidem intra ambitum paradysi terra absorbentur, sed longe in aliis regionibus emergunt. Nam Physon, qui et Ganges dicitur in India de monte Orcobares oritur et contra orientem fluens occeano Orientali excipitur. Erinnert sei in diesem Zusammenhang auch an die Flussfahrt im ,Herzog Ernst B', auf der der Herzog auf einem Floß durch eine dunkle Höhle kommt, in der er zwischen vielen anderen einen besonderen Edelstein findet, der im Dunkeln leuchtet - den sogenannten „Waisen“. Von ihm heißt es: der stein gap vil liehten glast, zitiert nach: Herzog Ernst. Ein mittelalterliches Abenteuerbuch, in der mittelhochdeutschen Fassung B nach der Ausgabe von Karl BARTSCH mit den Bruchstücken der Fassung A, hrsg. v. Bernhard SowinsKI (Universal-Bibliothek 8352), Stuttgart 2009, V. 4459. SowINSKI meint im Kommentar der Ausgabe, s. ebd., S. 390, dazu: ,Wegen der ihm zugesprochenen Leuchtkraft wird er in anderen Versionen und in der Hs. $b$ der Herzog-Ernst-Dichtung mit dem Karfunkel, der im Dunkeln leuchte, verwechselt." Und doch: Die mittelhochdeutsche Beschreibung klingt sehr nach dem splendidus carbunculus, den Ambrosius in ,De paradiso', s. SchenkL (Anm. 63), III, 15, für den Phison erwähnt und von dem Isidor sagt: Carbunculus autem dictus quod sit ignitus ut carbo, cuius fulgor nec nocte vincitur; lucet enim in tenebris adeo ut flammas ad oculos vibret, zitiert nach: Isidor von Sevilla, Etymologiarum sive originum libri XX, hrsg. v. Wallace M. LINDSAY (Scriptorum classicorum bibliotheca Oxoniensis), Bd. 2, Oxford 1911, ND Oxford 1957, XVI, 14, 1. 
schaften des Meeres und ihre symbolischen Ausdeutungen, wie sie im Brandanstoff anzutreffen sind, finden sich bereits im ,Hexaemeron' des Ambrosius nebeneinandergestellt - ich zitiere die Zusammenfassung nach REINITZER: „[E]inmal ist Meer der Lebensraum der Fische (Menschen), dann wieder lebensbedrohender Feind; einmal gibt es festen Halt, dann jedoch verschlingt und mordet es, bedeutet Strafe und Untergang; einmal reinigt es und wäscht Sünden ab, andererseits ist es selber unrein und sündenhaft. “70 Für die verschiedenen Bearbeitungen des Brandanstoffes konnten in der Betonung bzw. Vernachlässigung einzelner Charakteristika Akzentverschiebungen festgestellt werden. Diese gründen vor allem in der unterschiedlichen Figurenzeichnung Brandans, welche ein jeweils differierendes Zielpublikum im Blick hat. Andere Ausformungen des Wassers, die im Brandan-Corpus reichlich zu finden sind, sowie deren symbolische Bedeutungen konnten nur schlaglichtartig in den Fokus rücken. Insgesamt stellt eine auf das Wasser und seine Symbolik zentrierte Untersuchung im Brandan-Corpus nach wie vor ein Forschungsdesiderat dar. ${ }^{71}$ In diesem Zusammenhang müsste auch der Frage nach keltischen Einflüssen, wie sie sich beispielsweise in der Verortung des irdischen Paradieses im Westen spiegeln, noch intensiver nachgegangen werden.

70 Reinitzer (Anm. 11), S. 104.

71 Dieses Desiderat versuche ich in meinem Dissertationsprojekt zum Heiligen Brandan zu beseitigen. 